\title{
Successful Treatment of Atopic Dermatitis with Dupilumab in a Patient with Non-Hodgkin's Lymphoma
}

YunMi QIU ${ }^{1 *}$, Kamran ALI ${ }^{1 *}$, HaiYue LOU ${ }^{1}$, JingPeng SHAN ${ }^{1}$ and Liming WU2*

${ }^{1}$ Department of Dermatology, Zhejiang Chinese Medical University, Hangzhou, Zhejiang, and 2Department of Dermatology, Affiliated Hangzhou First People's Hospital, Zhejiang University School of Medicine, No.261, Huansha Road, Hangzhou, P. R. China *E-mail: limingwu1973@163.com \#These authors contributed equally to this work and share first authorship.

Accepted Dec 14, 2021; Epub ahead of print Dec 14, 2021

In recent years, biological agents have been widely used in dermatology. Practical issues, including comorbidities and tolerability, must be considered when choosing biological agents in clinical practice. Dupilumab is the first fully human monoclonal antibody licensed for use in atopic dermatitis (AD), and has been shown to have favourable efficacy and safety profile in AD treatment $(1,2)$. Dupilumab suppresses type 2 inflammation by inhibiting interleukin (IL)-4 and IL-13 cytokine signalling. No increased malignancy events have been reported associating with modulation of the IL-13/IL-4 pathways (3). A previous case report found that dupilumab treatment safely improved AD in 2 patients with cancer (melanoma and anal squamous cell carcinoma, respectively) without evidence of cancer recurrence (4). We present here a patient with non-Hodgkin's lymphoma with $\mathrm{AD}$ who showed a complete response to dupilumab therapy.

\section{CASE REPORT}

A 56-year-old woman with a 7-year history of AD presented with xerosis, pruritus, and relapsing eczematous eruptions, which were recalcitrant to topical treatment. Six years previously, she had had non-Hodgkin's lymphoma (NHL). After receiving lymph node biopsies, she was diagnosed with mucosa-associated lymphoid tissue (MALT) lymphoma, an extranodal marginal zone lymphoma, stage IIA, without bone marrow and other organs being affected. The patient was treated with 6 cycles of rituximab (MabThera, anti-CD20mAb, Roche Pharma, Switzerland) and achieved sustained complete remission. However, NHL recurred 1 year later and she received 9 continuous cycles of rituximab, achieving complete remission. In the year prior to presentation, the patient developed red patches on dry, cracked, and scaly skin with lichenification and prurigo nodules on her arms, abdomen, and legs (Fig. 1a). The Scoring Atopic Dermatitis (SCORAD) index was 57.5 (severe), Eczema Area and Severity Index (EASI) was 31.4, and Pruritus-numerical rating scale (Pruritus-NRS) $10 / 10$. Laboratory evaluations were in the normal range (IgE 21.9 kIU/1, blood eosinophil count: $\left.0.14 * 10^{9} / 1\right)$. A skin biopsy showed non-specific hyperkeratosis skin inflammation (Fig. 2). Immunohistochemistry findings revealed a few scattered $\mathrm{CD}^{+} \mathrm{T}$ cells, $\mathrm{CD} 20^{+} \mathrm{B}$ cells, $\mathrm{CD}^{+} \mathrm{T}$ cells. PAX 5 was negative, and approximately $10 \%$ of lymphocytes were $\mathrm{Ki}-67^{+}$. According to her medical history and these results, she was diagnosed with AD, based on the criteria of Hanifin \& Rajka differentiating it from cutaneous lymphoma. The patient was treated with topical medications, including mometasone furoate $0.1 \%$ cream, halometasone cream, and tacrolimus $0.1 \%$ ointment concomitant with antihistamines (Ebastine, KESTINE, Industrias Farmaceuticas Almirall, S.A., Sant Andreu de la Barca, Spain). However, the regimen failed

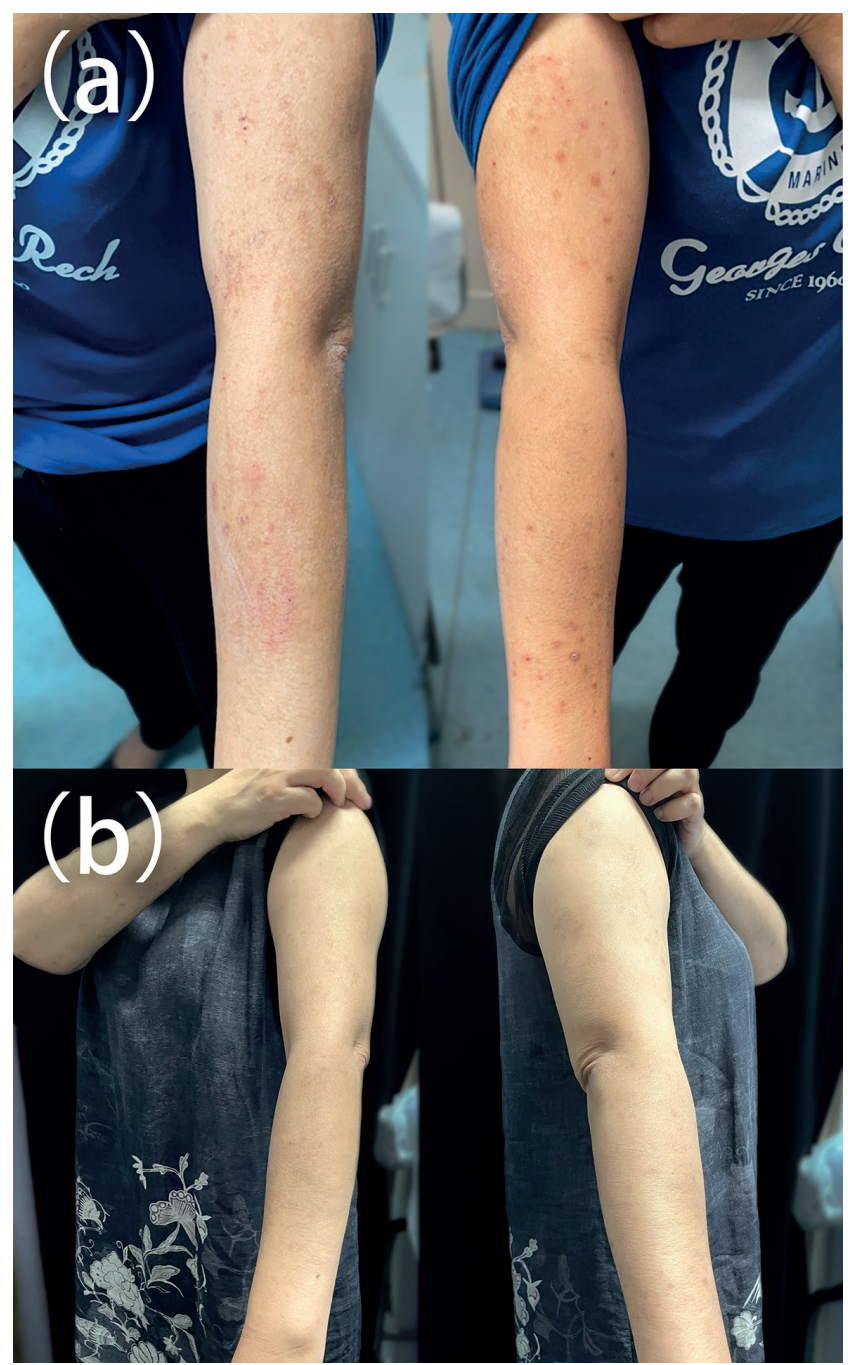

Fig. 1. (a) Dry, cracked, scaly skin with diffuse lichenified papules and nodules was visible on the arms prior to dupilumab treatment. (b) Total clearance of lesions after 5 months of dupilumab treatment.

to improve her condition. Systemic immunosuppressors were not considered because of safety concerns in cancer patients (5). Dupilumab was initiated at a $600 \mathrm{mg}$ loading dose, followed by $300 \mathrm{mg}$ biweekly. One month later, a significant decrease in severity, and improvement of pruritus-NRS 3/10, was observed. After 5 months of dupilumab therapy, the patient showed remarkable numerical improvement (SCORAD 12.2, EASI 3.4, pruritus-NRS 0/10), and no side-effects were observed. Physical examination showed only xerosis and pigmentation on her upper limbs (Fig. 1b). In addition, at follow-up in the Department of Haematology, the patient reported a stable condition, without recurrence of NHL. 


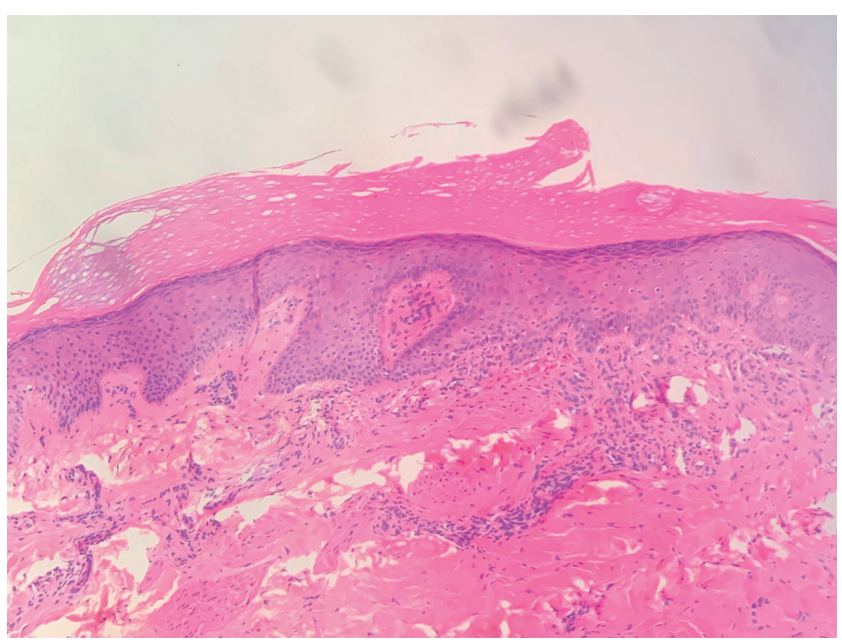

Fig. 2. A skin biopsy from the left mid thigh revealed non-specific skin inflammation: hyperkeratosis, mild irregular hypertrophy of epidermis, infiltration of lymphocytes around blood vessels in the superficial dermis (Haematoxylin \& Eosin staining; $\times 40$ )

\section{DISCUSSION}

To achieve a targeted efficacy profile in patients with $\mathrm{AD}$, biologic treatments are projected to replace topical corticosteroids and systemic immunosuppressants. Since 2020, the National Medical Products Administration (NMPA) has approved dupilumab for use in China for moderate-to-severe AD. Continuous dupilumab treatment has shown a long-term safety and efficacy profile in patients with moderate-to-severe AD (1). To our knowledge, this is the first published case of dupilumab treatment of severe AD in a patient with NHL. Before starting the treatment regimen, a skin biopsy was taken cautiously to define the diagnosis, since cutaneous T-cell lymphoma (CTCL), including mycosis fungoides and Sézary syndrome, present a similar clinical picture to that of AD. Experts suspect eczematous skin lesions may occur as a symptom of undiagnosed CTCL, especially in adult patients with late-onset AD (6). Some studies have described the progression of CTCL after receiving dupilumab therapy for presumed atopic dermatitis $(7,8)$. However, systemic immunosuppressors were not considered, due to the potential to cause lymphoproliferative diseases and malignancies. In a previously reported case, a patient with $\mathrm{AD}$ who had been treated with cyclosporine A developed non-Hodgkin's lymphoma (9). In the current case report, the patient was diagnosed with $\mathrm{AD}$ and rapidly experienced substantial improvements in itch severity, and was treated successfully with dupilumab without side-effects.

Moreover, $\mathrm{AD}$ has been found to be connected with an increased risk of NHL, and the risk correlated positively with the severity of eczema (10). It is promising that the current patient benefitted from dupilumab therapy. However, the long-term safety data for use of dupilumab in patients with cancer is insufficient and further studies are necessary.

\section{ACKNOWLEDGEMENTS}

Written informed consent for publication of personal details was provided by the patient.

The authors have no conflicts of interest to declare.

\section{REFERENCES}

1. Deleuran M, Thaçi D, Beck LA, de Bruin-Weller M, Blauvelt A, Forman S, et al. Dupilumab shows long-term safety and efficacy in patients with moderate to severe atopic dermatitis enrolled in a phase 3 open-label extension study. J Am Acad Dermatol 2020; 82: 377-388.

2. Chun PIF, Lehman H. Current and future monoclonal antibodies in the treatment of atopic dermatitis. Clin Rev Allergy Immunol 2020; 59: 208-219.

3. Braddock M, Hanania NA, Sharafkhaneh A, Colice G, Carlsson $M$. Potential risks related to modulating interleukin-13 and interleukin-4 signalling: a systematic review. Drug Saf 2018; 41: 489-509.

4. Fowler E, Rosen J, Lev-Tov H, Yosipovitch G. Two cancer patients receiving dupilumab for treatment of atopic dermatitis. Acta Derm Venereol 2019; 99: 899-900.

5. Arellano FM, Arana A, Wentworth CE, Fernández-Vidaurre C, Schlienger RG, Conde E. Lymphoma among patients with atopic dermatitis and/or treated with topical immunosuppressants in the United Kingdom. J Allergy Clin Immunol 2009; 123: 1111-1116.

6. Francuzik W, Alexiou A, Worm M. Safety of dupilumab in patients with atopic dermatitis: expert opinion. Expert Opin Drug Saf 2021; 20: 997-1004.

7. Miyashiro D, Vivarelli AG, Goncalves F, Cury-Martins J, Sanches JA. Progression of mycosis fungoides after treatment with dupilumab: a case report. Dermatol Ther 2020; 33: e13880.

8. Espinosa ML, Nguyen MT, Aguirre AS, Martinez-Escala ME, Kim J, Walker CJ, et al. Progression of cutaneous T-cell lymphoma after dupilumab: case review of 7 patients. J Am Acad Dermatol 2020; 83: 197-199.

9. Sinha A, Velangi S, Natarajan S. Non-Hodgkin's Iymphoma following treatment of atopic eczema with cyclosporin A. Acta Derm Venereol 2004; 84: 327-328.

10. Mansfield KE, Schmidt SAJ, Darvalics B, Mulick A, Abuabara $\mathrm{K}$, Wong AYS, et al. Association between atopic eczema and cancer in England and Denmark. JAMA Dermatol 2020; 156: 1086-1097. 